\title{
ILIACUS HAEMATOMA
}

\author{
Sadhan Kumar Mukherjee, Glasgow, Scotland \\ From the Orthopaedic and Accident Division, Glasgow Royal Infirmary
}

This paper reports two cases of iliacus haematoma complicating closed injury.

Case 1-A boy aged nine years was admitted in August 1969 with difficulty in walking and pain around the left hip after a fall from a wall three weeks before, in which he had injured the left side of the lower abdomen. The symptoms gradually worsened and he developed increasing flexion deformity of the left hip. There was no history of gastro-intestinal or urinary symptoms. On examination the child was obviously in pain and tended to lie curled on his side. The temperature was 36.4 degrees Celsius (97.5 degrees Fahrenheit) and the pulse 90 a minute. There was a mass in the left iliac fossa which was acutely tender. Bowel sounds were normal. Examination of the spine revealed slight tenderness over the left sacro-iliac joint, but movements were normal. There was a 60 degrees flexion deformity of the left hip with slight lateral rotation, but the hip could be actively flexed to 90 degrees. Other movements were only slightly restricted, by pain. Radiographs of the lumbar spine, pelvis and chest failed to show any abnormality. Intravenous pyelography was normal.

Investigations showed haemoglobin to be $11 \cdot 1$ grammes per 100 millilitres, and the white cell count to be 8,200 per cubic millimetre. There was anisocytosis with variation in haemoglobin; rouleaux formation was noted; neutrophils showed toxic granulation; platelets were present. The erythrocyte sedimentation rate was 80 millimetres in the first hour. Blood culture did not yield any organism. The Mantoux test was negative.

Treatment and progress - Treatment was initially by bed rest until the sixth day, when the patient developed pyrexia of 38.8 degrees Celsius (101.8 degrees Fahrenheit). At this point cloxacillin and penicillin were begun parenterally. Attempted aspiration of the left iliac fossa under local anaesthesia was unsuccessful.

On the eighth day the patient was examined under anaesthesia. Abdominal examination revealed a hard mass filling the whole of the left iliac fossa and extending from just above the iliac crest to the inguinal ligament. Rectal examination did not reveal any abnormality apart from a loaded rectum. There was a $\mathbf{4 5}$ degrees fixed flexion deformity of the left hip: rotation was full. Under the anaesthetic aspiration of the mass in the left iliac fossa was carried out with a wide bore needle: 150 millilitres of fluid-pus mixed with blood and clot-were aspirated. Bacteriological examination of the fluid showed numerous pus cells and grampositive cocci, mainly in clusters, but a few in chains. Acid-fast bacilli were not seen. Culture yielded a heavy growth of staphylococcus aureus and a light growth of $B$ haemolytic streptococci (Lancefield Group A).

Cloxacillin-to which both organisms were sensitive-was continued, but the patient's condition continued to deteriorate.

Operation-At operation two weeks after the patient's admission and five weeks after the injury the abdomen was opened through a left paramedian incision. The pelvic colon was adherent to the mass but was normal, as were the other abdominal viscera. The psoas tendon ran through the posterior part of the tough fibrous wall of the haematoma, this accounting for the flexion deformity of the left hip. The wound was closed with drainage. It was thought likely that the haematoma in the iliac fossa was a consequence of the injury, and that it subsequently became infected, tying the pelvic colon to the haematoma.

Further progress-Antibiotic therapy was continued and the patient made steady but slow progress towards full recovery. Subsequent investigation failed to show any evidence of blood dyscrasia. 
Case 2-A man of twenty-four was admitted in January 1970 complaining of difficulty in walking and pain in the left groin after twisting the limb two weeks previously. The symptoms gradually worsened and at the time of admission he had developed a flexion deformity of the left hip joint and was barely able to walk. On examination the patient's general condition was good, with a pulse rate of 74 per minute and temperature of 37.2 degrees Celsius ( 99 degrees Fahrenheit). Abdominal examination showed an ill-defined but very tender mass in the left iliac fossa. The spine showed a moderate degree of lumbar lordosis. There was a very tender swelling in the left femoral triangle and adjoining part of the left iliac fossa. There was a 90-degree flexion deformity and a slight lateral rotation deformity of the left hip. Attempts at passive correction of the flexion deformity produced intense pain, but full flexion of the hip joint beyond 90 degrees could be achieved passively. Active flexion caused pain. Passive rotation, abduction and adduction were restricted because of pain.

Investigations-Examination of the blood showed haemoglobin of 14.5 grammes per 100 millilitres; there was a neutrophil leucocytosis with total white cell count of 27,100 , and the erythrocyte sedimentation rate was 70 millimetres in one hour.

Radiographs of the chest, lumbar spine and left hip showed no abnormality.

Treatment and progress-The patient was treated initially by bed rest, analgesics and bilateral leg traction. However, he started having intermittent pyrexia of up to 39.2 degrees Celsius (102.6 degrees Fahrenheit) daily.

Four days after admission the swelling in the left groin had increased in size and had become fluctuant. At examination under anaesthesia the left hip joint could be extended fully and other movements were almost full. The swelling in the left groin was aspirated and 70 millilitres of pus were aspirated. Pus cells and gram-positive cocci were seen in this material, and culture yielded a moderate growth of staphylococcus aureus sensitive to cloxacillin and tetracycline but resistant to penicillin and ampicillin.

Cloxacillin ( 2 grammes 6-hourly by injection) was started, but pyrexia continued and the swelling in the groin reappeared and became larger.

Operation-A week after admission an incision was made over the prominent part of the swelling below the inguinal ligament. About 0.4 litre of pus mixed with altered blood was released after sustained pressure on left iliac fossa. A drain was inserted.

The patient's progress was good, and by the end of February the wound was healed; there was no residual collection of pus and movements of the hip were full. There were, however, weakness and wasting of the quadriceps muscle. Eight months after operation the patient reported that he had no symptoms and was fully active.

\section{DISCUSSION}

Iliacus haematoma is a collection of blood in the iliacus muscle-that is, in a closed compartment bounded anteriorly by the fascia covering the muscle and posteriorly by the ilium. It is rare, but it may occur in patients with haemophilia or as a complication of anticoagulant therapy. It has also been reported in individuals with blood dyscrasia since the original publication of Getting's case by Bulloch and Fildes in 1911. It is extremely unusual after injury in a patient with a normal blood picture. Fisher (1968) reported one instance of traumatic iliacus haematoma in a boy of sixteen who had a comminuted fracture of the anterior superior iliac spine, but I have been unable to find any reference to such a complication arising from closed trauma, without bony injury, in an otherwise normal individual.

The presenting symptom is pain in the groin, spreading to the lumbar region or thigh, and steadily increasing until it is severe and constant. The hip on the affected side is held in flexion and slight lateral rotation. Extension of the hip makes the pain worse. A very tender mass is always present in the iliac fossa. Femoral nerve palsy develops in most cases. 
The syndrome is relatively common in patients suffering from blood dyscrasia. The first instance of this was Getting s case reported by Bulloch and Fildes (1911) in a patient with haemophilia. Tallroth (1939) reported a case in which large spontaneous haemorrhages in the iliopsoas compartment were noted on two occasions. Davidson, Epstein, Miller and Taylor (1949) reported fifteen cases of iliacus haematoma in a series of forty haemophiliac patients. Goodfellow, Fearn and Matthews (1967) reported twenty-four cases of iliacus haematoma in haemophiliac patients. They described the clinical condition and showed by an injection experiment on a cadaver how pressure on the femoral nerve was produced by overflow of fluid from the iliacus compartment. The femoral nerve was clearly affected in the second of the two cases described here. In both cases reported here the haematomata were infected by staphylococcus aureus, and in both the classical syndrome appeared relatively late. The haematomata were presumably produced by rupture of muscle fibres at the time of the injury.

The experience gained from these two cases suggests that iliacus haematoma in healthy individuals should be drained by operation once the diagnosis is made, in order to avoid infection and the possible consequence of fixed flexion contracture of the hip.

\section{SUMMARY}

1. Two cases of iliacus haematoma occurring after injury in otherwise healthy individuals are reported.

2. Both cases were complicated by infection of the haematoma, but both patients made a full recovery after operation.

I am grateful to $\mathrm{Mr}$ S. Alexander and Mr K. Protheroe for allowing me to report their cases. I would like to thank Mr J. White and Mr A. B. Young for their help in the preparation of this paper.

\section{REFERENCES}

Bulloch, W., and Fildes, P. (1911): Haemophilia. (Eugenics Laboratory Memoirs, 12). Treasury of Human Inheritance, Parts V and VI, Section XIVa.

Davidson, C. S., Epstein, R. D., Miller, G. F., and Taylor, F. H. L. (1949): Haemophilia: A Clinical Study of Forty Patients. Blood, 4, 97.

Fisher, T. R. (1968): Iliacus Haematoma and Nerve Palsy. British Medical Journal, 4, 391.

Goodfellow, J., Fearn, C. B. d’A., and Matthews, J. M. (1967): Iliacus Haematoma: A Common Complication of Haemophilia. Journal of Bone and Joint Surgery, 49-B, 748.

TAllroth, A. (1939): Hemophilia with Spontaneous Hemorrhage in the Iliopsoas Muscle Followed by Injury to the Femoral Nerve. Acta Chirurgica Scandinavica, 82, 1.

VOL. $53 \mathrm{~B}$, NO. 4, NOVEMBER 1971 